\title{
MINIMAL FINE DERIVATIVES AND BROWNIAN EXCURSIONS
}

\author{
KRZYSZTOF BURDZY
}

\section{$\S 1$. Introduction}

The paper will present some basic properties of minimal fine derivatives which seems to be a new concept (or at least a new combination of wellknown ones). Why is it worthwhile to study this new concept? Why hasn't it been done earlier?

As for the first question, it should be noted that:

i) The minimal fine convergence is better suited to the potential theory than the nontangential convergence which has been traditionally used in connection with the concept of the boundary derivative ("angular derivative"). See how natural, almost obvious, Theorem 3.2 sounds. An analogous result for angular derivatives (Jackson (1965)) may seem puzzling at the first sight.

ii) Many important theorems about existence of angular derivatives have their counterparts for minimal fine derivatives. Showing this will be the main goal of this paper. Since the minimal fine topology is stronger than the "nontangential topology" (in the sense of Lemma 2.2), several known theorems about angular derivatives follow immediately from the results derived here.

iii) Minimal fine derivatives are much more useful in probability than angular derivatives. The results of Section 4 could not have been obtainned with the help of angular derivatives.

As for the second question posed at the beginning, the reason for the neglect of the minimal fine derivatives may be the esoteric nature of the minimal fine neighborhoods. A simple characterization of simply connected minimal fine neighborhoods of a boundary point in halfplane will be given in Lemma 2.1. This characterization has appeared in Burdzy (1986) (Theorems 5.2 and 5.3). It should be pointed out, however, that it could have

Received August 9, 1988.

Research supported in part by NSF Grant DMS 8419377. 
been deduced from earlier results of Jackson (1965) and Rodin and Warschawski (1977), for example. A related paper on minimal fine sets by Essén and Jackson (1980) should be also mentioned here.

The present paper has been inspired by the article of Davis (1979).

It is impossible to give in the short "Preliminaries" section a thorough review of all the concepts used in the paper. Thus the reader is referred to Burdzy (1987), Doob (1984), Maisonneuve (1975), Naim (1957), Pommerenke (1975) and Rodin and Warschawski (1977) for more details.

\section{§ 2. Preliminaries}

The real and imaginary parts of $x$ will be denoted $\Re x$ and $\mathfrak{\Im} x$. The sets of all real and complex numbers will be denoted $\mathbb{R}$ and $\mathbb{C} . \mathbb{R}_{+}=$ $\{x \in \mathbb{R}: x>0\}=\{x \in \mathbb{C}: \mathfrak{\Im} x=0, \Re x>0\}, D_{*}=\{x \in \mathbb{C}: \Re x>0\}, S(\alpha)=$ $\left\{x \in D_{*}:|\arg x|<\alpha\right\} . \quad S(\alpha)$ is called a Stolz angle. For $D \subset \mathbb{C}$, the notation $f: D \rightarrow D_{1}$ will indicate that $f$ is an analytic function, one-to-one and onto $D_{1}$.

"Lipscnitz function" will mean a function with the Lipschitz constant equal to 1 i.e., a function $f$ satisfying $|f(x)-f(y)| \leq|x-y|$ for all $x$ and $y$.

The Green function of a Greenian set $D \subset \mathbb{C}$ will be denoted $G_{D}$. $K_{D}^{x}(y, z) \stackrel{\text { def }}{=} G_{D}(y, z) / G_{D}(y, x)$ will be called the Martin function $(y \neq x)$. There exists a unique up to a homeomorphism compactification $D^{M}$ of $D$ such that $K_{D}^{x}$ may be extended continuously to $D^{M} \backslash\{x\} \times D$. It will be called the Martin compactification and $\partial^{M} D \stackrel{\text { def }}{=} D^{M} \backslash D$ will be called the Martin boundary of $D$. For simply connected plane domains, the Martin boundary $\partial^{M} D$, the minimal Martin boundary $\partial_{1}^{M} D$ and the Caratheodory prime end boundary coincide (see Doob (1984) and Pommerenke (1975) for definitions).

A set $A \subset D$ will be called minimal thin in $D$ at $y \in \partial_{1}^{M} D$ if $\lim _{z \rightarrow y} f(z) / K_{D}^{x}(y, z)=\infty$ for some superharmonic function $f$ in $D$. This defini$\underset{\substack{x \rightarrow y \\ z \in A}}{t \rightarrow 0}$

tiontells very little about properties of minimal thin sets. Doob (1984) and Naim (1957) are rich sources of relevant results. To define minimal fine topology, call a set $A \subset D$ a minimal fine neighborhood of $y \in \partial_{1}^{M} D$ if $D \backslash A$ is minimal thin in $D$ at $x$. The limits in the minimal fine topology will be denoted mf-lim.

The Green function, minimal thinness and minimal fine topology are 
conformal invariants.

Lemma 2.1. i) $A$ simply connected open set $D \subset D_{*}$ is a minimal fine neighborhood of 0 in $D_{*}$ if and only if there exist $\varepsilon>0$ and a nonnegative Lipschitz function $h: \mathbb{R} \rightarrow \mathbb{R}$ such that

$$
\int_{-1}^{1} h(r) r^{-2} d r<\infty \quad \text { and } \quad\{x \in \mathbb{C}: \Re x>h(\widetilde{\Im} x),|x|<\varepsilon\} \subset D .
$$

ii) If $D$ is a simply connected open set and a minimal fine neighoorhood of 0 in $D_{*}$ then for each $\alpha<\pi / 2$ there exists $\varepsilon>0$ such that $\{x \in S(\alpha)$; $|x|<\varepsilon\} \subset D$.

Proof. i) See Theorems 5.2 and 5.3 of Burdzy (1986).

ii) The Lipschitz property of $h$ and the finiteness of the integral $\int_{-1}^{1} h(r) r^{-2} d r$ imply easily that $\lim _{r \rightarrow 0} h(r) / r=0$. From this, the assertion follows immediately.

It will be said that a function $f$ defined on $D_{*}$ has the angular limit $a$ at $x \in \partial D_{*}$ if $\lim _{\substack{z \rightarrow x \\ z-x \in S(\alpha)}} f(z)=a$ for every $\alpha<\pi / 2$.

The following result is known (see e.g. Davis (1979)) but the method of proof seems to be new.

Lemma 2.2. Suppose that $f$ is analytic in $D_{*}$ and $z \in \partial D_{*}$. If $m f$-lim $\lim _{x \rightarrow z}$ $f(x)=a$ then the angular limit of $f$ at $z$ exists and equals $a$.

Proof. Assume without loss of generality that $z=0$. Choose $\varepsilon>0$ and let $A=\left\{x \in D_{*}:|f(x)-a|<\varepsilon\right\}$. The set $A$ is simply connected by the maximum principle. Lemma 2.1 (ii) implies that for every $\alpha<\pi / 2$ there exists $\varepsilon_{1}>0$ such that $\left\{x \in S(\alpha):|x|<\varepsilon_{1}\right\} \subset A$. This implies that the angular limit of $f$ at 0 is $a$, since $\varepsilon>0$ is arbitrary.

Suppose that $f: D_{*} \rightarrow D$ and $y \in \partial D_{*}$. If the limit of $f(x)$ exists for $x \rightarrow y$ along a single continuous line in $D_{*}$ then it exists in the minimal fine topology (Jackson (1980)). By Lemma 2.2, it exists as an angular limit as well. The common value of the limits will be denoted $f(y)$, provided they exist. If $0 \in \partial D$ and $\left\{x \in \mathbb{R}_{+}:|x|<\varepsilon\right\} \subset D$ for some $\varepsilon>0$ then this line segment defines a prime end in $D$ which will be denoted $0^{M}$.

The domains considered in the paper (usually denoted by $D$ with a subscript) will be always assumed open and simply connected as subsets 
of the Riemann sphere.

Each time the function arg is used, a version of this function will be chosen so that it will be continuous on the set of all arguments appearing in the statement.

A review of probabilistic concepts is offered in Section 4.

\section{§3. Minimal fine derivatives}

Definition 3.1. For a function $f: D_{*} \rightarrow D$, the minimal fine (angular) limit of $f^{\prime}$ at $x \in \partial D_{*}$ will be called the minimal fine (angular) derivative of $f$ at $x$ and denoted $\mathrm{mf}-f^{\prime}(x)\left(\mathrm{a}-f^{\prime}(x)\right)$, provided it exists.

Proposition 3.1. If $\mathrm{mf}-f^{\prime}(x)=a$ then a- $f^{\prime}(x)=a$.

Proof. The function $f^{\prime}$ is analytic in $D_{*}$ so the result follows from Lemma 2.2.

The converse statement is false; see Example 3.1 below.

Theorem 3.1. If $f: D_{*} \rightarrow D, x \in \partial D_{*}$ and $\mathrm{mf}-f^{\prime}(x)=a \in \mathbb{C}$ then $f(x)$ exists and

$$
\operatorname{mf}-\lim _{\substack{z \rightarrow x \\ z \in D_{*}}}(f(z)-f(x)) /(z-x)=a
$$

Proof. Assume without loss of generality that $x=0$.

Choose an $\varepsilon>0$ and let $A=\left\{z \in \mathbb{C}:\left|f^{\prime}(z)-a\right|<\varepsilon\right\}$. The set $A$ is simply connected by the maximum principle and it is a minimal fine neighborhood of 0 in $D_{*}$, by the assumption that $m f-f^{\prime}(0)=a$. According to Lemma 2.1, one can choose a nonnegative Lipschitz function $h: \mathbb{R} \rightarrow \mathbb{R}$ and $\varepsilon_{1}>0$ such that

$$
B \stackrel{\text { def }}{=}\left\{z \in \mathbb{C}: \Re z>h(\mathfrak{\Im} z),|\Re z| \leq \varepsilon_{1},|\mathfrak{s} z|<\varepsilon_{1}\right\} \subset A
$$

and $B$ is a minimal fine neighborhood of 0 in $D_{*}$. Some initial segment $I$ of $\mathbb{R}_{+}$is contained in $B$. Since $f^{\prime}$ is continuous and bounded on $I$, $\lim f(z)$ exists. It may be assumed without loss of generality that this $\underset{\substack{z \rightarrow 0 \\ z \in I}}{\lim }$

limit is equal to 0 , i.e. $f(0)=0$.

For each $z \in B$ choose a polygonal line $\Gamma$ in $B$ with endpoints $z$ and 0 , such that its length is not greater than $3|z|$ and it approaches 0 along $\mathbb{R}_{+}$. In particular, $\lim _{\substack{z \in 0 \\ z \in \Gamma}} f(z)=0$. One has 


$$
\begin{aligned}
|f(z)-a z| & =\left|\int_{\Gamma} f^{\prime}(z) d z-a z\right| \\
& =\left|\int_{\Gamma} a d z+\int_{\Gamma}\left(f^{\prime}(z)-a\right) d z-a z\right| \\
& =\left|\int_{\Gamma}\left(f^{\prime}(z)-a\right) d z\right| \\
& \leq \int_{\Gamma}\left|f^{\prime}(z)-a\right| d z \leq \varepsilon \cdot 3|z|
\end{aligned}
$$

for $z \in B$. Thus

$$
\limsup _{\substack{z \rightarrow 0 \\ z \in B}}\left|\frac{f(z)-f(0)}{z-0}-a\right|=\limsup _{\substack{z \rightarrow 0 \\ z \in B}}\left|\frac{f(z)}{z}-a\right| \leq 3 \varepsilon .
$$

Since $\varepsilon>0$ is arbitrary and $B=B(\varepsilon)$ is a minimal fine neighborhood of 0 in $D_{*}$, mf- $\lim _{\substack{z \rightarrow 0 \\ z \in D_{*}}}(f(z)-f(0)) /(z-0)=a$.

Remark 3.1. i) The existence of $f(x)$ and $\operatorname{mf}-\lim _{\substack{z \rightarrow x \\ z \in D_{*}}}(f(z)-f(x)) /(z-x)$ does not guarantee that $\mathrm{mf}-f^{\prime}(x)$ exists. A counterexample may be constructed along the lines of Example 9.2 of Burdzy (1987). Such a construction is extremely long and therefore it is omitted here.

ii) Both the theorem and its converse are true for angular derivatives (Pommerenke (1975) Theorem 10.5).

iii) Later on, the following two simple facts about functions of the form $f: D_{*} \rightarrow D$ will be used. If $\operatorname{mf}-\lim _{x \rightarrow z} f(x)=0^{M}$ then $f(z)=0$. If $\mathrm{mf}$ $f^{\prime}(z) \in \mathbb{R}_{+}$and $f(z)=0$ then $\operatorname{mf}_{x \rightarrow z} f(x)=\lim _{x}$. The last statement is a consequence of Theorem 3.1 and Lemma 2.2.

ExAmple 3.1. Example 9.1 of Burdzy (1987) presents a domain $D$ with the following properties.

i) There exists a function $f: D_{*} \rightarrow D$ such that $f(0)=0$ and a- $f^{\prime}(0)=$ $a \in \mathbb{R}_{+}$.

ii) The sets $A_{1} \stackrel{\text { df }}{=}\left\{z \in D_{*}: \arg f(z)>\pi\right\}$ and $A_{2} \stackrel{\text { df }}{=}\left\{z \in D_{*}: \arg f(z)\right.$ $<-\pi\}$ are not minimal thin in $D_{*}$ at 0 .

Suppose that mf- $f^{\prime}(0)$ exists. Then $m f-f^{\prime}(0)=a$ by Proposition 3.1 and $\mathrm{mf}-\lim f(z) / z=a$ by Theorem 3.1. It follows that

$\underset{\substack{z \rightarrow 0 \\ z \in D_{*}}}{ }$

$$
\operatorname{mf}-\lim _{\substack{z \rightarrow 0 \\ z \in D_{*}}}(\arg f(z)-\arg z)=\arg a
$$

and 


$$
A_{3} \stackrel{\text { df }}{=}\left\{z \in D_{*}:|\arg f(z)-\arg z-\arg a|<\varepsilon\right\}
$$

is a minimal fine neighborhood of 0 in $D_{*}$, for every $\varepsilon>0$. At least one set $A_{1}$ or $A_{2}$ lies in the complement of $A_{3}$, for small $\varepsilon>0$. This is a contradiction since $A_{1}$ and $A_{2}$ are not minimal thin in $D_{*}$ at 0 .

It may be concluded that $\mathrm{mf}-f^{\prime}(x)$ does not exist although a- $f^{\prime}(x)$ exists.

Definition 3.2. It will be said that a set $D \subset \mathbb{C}$ has a minimal fine derivative if there eixsts a function $f: D_{*} \rightarrow D$ such that mf- $f^{\prime}(0) \in \mathbb{R}_{+}$ and $f(0)=0$.

Problem 3.1 ("Minimal fine derivative problem"). Find necessary and sufficient conditions of geometric nature for $D$ so that it has a minimal fine derivative.

REMARK 3.2. The above problem is analogous to the well known "angular derivative problem". See Pommerenke (1975) and Rodin and Warschawski (1977) for the history of that problem.

Theorem 3.2. A domain $D \subset D_{*}$ has a minimal fine derivative if and only if it is a minimal fine neighborhood of 0 in $D_{*}$.

Proof. i) Suppose that $D$ has a minimal fine derivative i.e., there exists a function $f: D_{*} \rightarrow D$ such that $f(0)=0$ and $m f-f^{\prime}(0) \in \mathbb{R}_{+}$. Then a- $f^{\prime}(0) \in \mathbb{R}_{+}$, by Proposition 3.1. Theorem 5.2 of Burdzy (1986) implies that $D_{*} \backslash D$ is minimal thin in $D$ at 0 .

ii) Assume that $D$ is a minimal fine neighborhood of 0 in $D_{*}$ and fix some $x \in D$. Since $D$ is simply connected and open (a tacit assumption introducced in Section 2), it follows easily from Lemma 2.1 that the prime end $0^{M}$ is well defined. The limits

$$
\operatorname{mf}-\lim _{\substack{z \rightarrow M M \\ z \in D}} G_{D_{*}}(z, x) / G_{D}(z, x)
$$

and

$$
\limsup _{\substack{z \rightarrow 0 M \\ z \in D}} G_{D_{*}}(z, x) / G_{D}(z, x)
$$

exist and are equal by Theorem 1 XII 14 of Doob (1984). Theorem 11 of Naim (1957) shows that they are equal to some $a \in \mathbb{R}_{+}$.

Explicit formulae for the Green function given in Section 1 XII 9 of Doob (1984) yield 


$$
\lim _{\substack{z \rightarrow 0 \\ z \in D_{*}}} G_{D_{*}}(z, y) / \Re z=c=c(y) \in \mathbb{R}_{+} .
$$
Choose a function $f: D_{*} \rightarrow D$ such that $\operatorname{mf}-\lim _{\substack{z \in 0 \\ z \in D_{*}}} f(z)=0^{M}$. Then $f(0)$

$=0$. Recall that the Green function and minimal fine topology are conformal invariants and use this fact together with (3.1) and (3.2) to obtain

$$
\begin{aligned}
\operatorname{mf}-\lim _{\substack{z \rightarrow 0 \\
z \in D_{*}}} \Re f(z) / \Re z & =\operatorname{mf}-\lim _{\substack{z \rightarrow 0 \\
z \in D_{*}}} \Re f(z) c\left(f^{-1}(x)\right) / G_{D_{*}}\left(z, f^{-1}(x)\right) \\
& =\operatorname{mf}-\lim _{\substack{z \rightarrow 0 \\
z \in D_{*}}} \Re f(z) c\left(f^{-1}(x)\right) / G_{D}(f(z), x) \\
& =\operatorname{mf}-\lim _{\substack{z \rightarrow 0 \\
z \in D_{*}}} G_{D_{*}}(f(z), x) c\left(f^{-1}(x)\right) /\left[G_{D}(f(z), x) c(x)\right] \\
& =\operatorname{mf}-\lim _{\substack{v \rightarrow 0 M \\
v \in D}} G_{D_{*}}(v, x) c\left(f^{-1}(x)\right) /\left[G_{D}(v, x) c(x)\right] \\
& =a c\left(f^{-1}(x)\right) / c(x) \stackrel{\mathrm{df}}{=} b \in \mathbb{R}_{+} .
\end{aligned}
$$

Let $A(\varepsilon)=\left\{z \in D_{*}:|\Re f(z) / \Re z-b|<\varepsilon\right\}$ for $\varepsilon>0$.

The set $A(\varepsilon)$ is simply connected. To see this, suppose that there exists a point $y \notin A(\varepsilon)$ and a closed Jordan $\operatorname{arc} \Gamma \subset A(\varepsilon)$ around $y$. Let $\mu$ be the harmonic measure on $\Gamma$ relative to $y$, in the domain containing $y$ and bounded by $\Gamma$. The functions $\Re f(z)$ and $\mathfrak{R} z$ are positive and harmonic in $D_{*}$ so

$$
\Re f(y)=\int_{\Gamma} \Re f(z) \mu(d z)<\int_{\Gamma}(b+\varepsilon) \Re z \mu(d z)=(b+\varepsilon) \Re y .
$$

The strict inequality between the integrals follows from the continuity of the integrands. Thus $\Re f(y) / \Re y-b<\varepsilon$ and analogously $\mathfrak{R} f(y) / \mathfrak{R} y-b>$ $-\varepsilon$, contrary to the assumption that $y \notin A(\varepsilon)$. This contradiction shows that $A(\varepsilon)$ is simply connected.

Each set $A(\varepsilon)$ is a minimal fine neighborhood of 0 in $D_{*}$, by (3.3). Use Lemma 2.1 to find nonnegative Lipschitz functions $h_{\varepsilon}: \mathbb{R} \rightarrow \mathbb{R}$ such that

$$
\left\{z \in \mathbb{C}: \mathfrak{R} z>h_{\varepsilon}(\mathfrak{\Im} z),|z|<\alpha_{\varepsilon}\right\} \subset A(\varepsilon)
$$

for some $\alpha_{\varepsilon}>0$ and $\int_{-1}^{1} h_{\varepsilon}(r) r^{-2} d r<\infty$. For each integer $k \leq 1$ find $\eta_{k}>0$ such that $\eta_{k}>\alpha_{2-k}$ and

$$
\int_{-\eta_{k}}^{\eta_{k}} h_{2-k}(r) r^{-2} d r<2^{-k}
$$


Let $h_{2-k}^{1}: \mathbb{R} \rightarrow \mathbb{R}$ be the largest Lipschitz function such that $h_{2-k}^{1}(r)=0$ for $|r| \geq \eta_{k}$ and $h_{2-k}^{1}(r) \leq h_{2-k}(r)$ for all $r$. It is easy to see that $h_{2-k}^{1}(r)=$ $h_{2-k}(r)$ for all $r$ in some neighborhood of 0 and therefore

$$
\left\{z \in \mathbb{C}: \Re z>h_{2-k}^{1}(\mathfrak{S} z),|z|<\beta_{k}\right\} \subset A\left(2^{-k}\right)
$$

for some $\beta_{k}>0$. Define $g=\sup _{k \geq 1} h_{2-k}^{1}$. The function $g$ is Lipschitz and

$$
\begin{aligned}
\int_{-1}^{1} g(r) r^{-2} d r & \leq \sum_{k=1}^{\infty} \int_{-1}^{1} h_{2-k}^{1}(r) r^{-2} d r \\
& \leq \sum_{k=1}^{\infty} \int_{-\eta_{k}}^{\eta_{k}} h_{2-k}(r) r^{-2} d r \\
& \leq \sum_{k=1}^{\infty} 2^{-k}=1 .
\end{aligned}
$$

Thus $B \stackrel{\text { df }}{=}\{z \in \mathbb{C}: \mathbb{R} z>g(\Re z)\}$ is a minimal fine neighborhood of 0 in $D_{*}$, by Lemma 2.1. Moreover $\left\{z \in B:|z|<\beta_{k}\right\} \subset A\left(2^{-k}\right)$ so

$$
\lim _{\substack{z \rightarrow 0 \\ z \in B}} \Re f(z) / \Re z=b .
$$

For each integer $k$ construct a Lipschitz function $g_{k}$ as follows.

Fix $k$ and assume without loss of generality that $g(r) \leq 1$ for all $r$. Let $Q$ be the family of all intervals $I$ with the following properties.

i) $I=\left[m 2^{-n},(m+1) 2^{-n}\right]$ for some integers $m$ and $n, n \geq 1, m \neq 0$, -1 ,

ii) $g(r) \geq 2^{-n+1}$ for some $r \in I$,

iii) $g(r)<2^{-s}$ for all integers $j, s, 0 \leq s<n-1$, such that $I \subset$ $\left[j 2^{-s-1},(j+1) 2^{-s-1}\right]$ and all $r \in\left[j 2^{-s-1},(j+1) 2^{-s-1}\right]$.

It is easy to see that the intervals in $Q$ are either disjoint or have one point in common and $g$ vanishes on $\mathbb{R} \backslash \bigcup_{Q} I$. Let $g_{k}^{I}$ be the smallest nonnegative Lipschitz function such that $g_{k}^{I}\left(m 2^{-n}\right)=2^{-n+2} k$ (here $I=$ $\left.\left[m 2^{-n},(m+1) 2^{-n}\right]\right)$.

Observe that $g(r) / r \rightarrow 0$ as $r \rightarrow 0$ so there exists $\gamma_{k}>0$ such that $k g(r) / r<1 / 4$ for $|r|<\gamma_{k}$. Let $J_{k}=\left[-\gamma_{k}, \gamma_{k}\right]$. Then $g_{k}^{I}(r)=0$ for $I \subset J_{k}$ and

$$
|r|< \begin{cases}m 2^{-n-1} & \text { if } m>0, \\ |m+1| 2^{-n-1} & \text { if } m<-1 .\end{cases}
$$

It follows that for $I \subset J_{k}$ and $m \geq 0$,

$$
\int_{\mathrm{R}} g_{k}^{I}(r) r^{-2} d r \leq \int_{m 2^{-n}-2^{-n+2 k}}^{m 2^{-n}+2-n+2 k} 2^{-n+2} k\left(m 2^{-n-1}\right)^{-2} d r=128 k^{2} m^{-2} .
$$


The Lipschitz property of $g$ implies that $\inf _{I} g(r) \geq 2^{-n}$ and therefore

$$
\int_{I} g(r) r^{-2} d r \geq \int_{m 2^{-n}}^{(m+1) 2-n} 2^{-n}\left[(m+1) 2^{-n}\right]^{-2} d r=(m+1)^{-2} .
$$

Thus

$$
\int_{\mathbf{R}} g_{k}^{I}(r) r^{-2} d r \leq 128 k^{2} m^{-2}(m+1)^{2} \int_{I} g(r) r^{-2} d r \leq 512 k^{2} \int_{I} g(r) r^{-2} d r
$$

for $I \subset J_{k}, m>0$. An analogous proof shows that the inequality holds also for $m<-1$.

Define $g_{k}=\sup _{\substack{I \subset J_{k} \\ I \in Q}} g_{j}^{I}$. Then $g_{k}$ is Lipschitz and

$$
\begin{aligned}
\int_{-1}^{1} g_{k}(r) r^{-2} d r & \leq \sum_{\substack{I \subset J_{k} \\
I \in Q}} \int_{\mathrm{R}} g_{k}^{I}(r) r^{-2} d r \\
& \leq \sum_{\substack{I \subset J_{k} \\
I \in Q}} 512 k^{2} \int_{I} g(r) r^{-2} d r \\
& \leq 512 k^{2} \int_{-\gamma_{k}}^{\gamma_{k}} g(r) r^{-2} d r<\infty
\end{aligned}
$$

Recall how $g$ has been constructed from $h_{2-k}$ 's. Repeat the same construction with $g_{k}$ 's to obtain a Lipschitz function $\tilde{g}: \mathbb{R} \rightarrow \mathbb{R}$ such that $\tilde{g} \geq 0$,

$$
\int_{-1}^{1} \tilde{g}(r) r^{-2} d r<\infty
$$

and for each $k \geq 1, \tilde{g}(r) \geq g_{k}(r)$ for all $r$ in some neighborhood of 0 (depending on $k$ ). Denote

$$
C=\left\{z \in D_{*}: \mathfrak{R} z>\tilde{g}(\mathfrak{J} z)\right\} .
$$

Elementary geometry shows that $|z-y| \geq \xi_{k} \Re z$ for all $z \in D_{*}$ such that $\Re z \geq g_{k}^{I}(\mathfrak{S} z)$ and $y \in D_{*}$ such that $\mathfrak{\Im} y \in I$, $\Re y \leq g(\mathfrak{s} y)$. The constants $\xi_{k}$ may be chosen so that $\lim _{k \rightarrow \infty} \xi_{k}=1$. It follows that $|z-y| \geq \xi_{k} \Re z$ for all $z \in D_{*}$ such that $\mathfrak{R} z \geq g_{k}(\mathfrak{J} z)$ and $y \in D_{*}$ such that $\mathfrak{\Im} y \in J_{k}$, $\Re y \leq g(\mathfrak{\Im} y)$. This in turn implies that $\operatorname{dist}(z, \partial B) \geq \xi_{k} \Re z$ for all $z \in C$ provided $|z|$ is sufficiently small. Thus

$$
\lim _{\substack{z \rightarrow 0 \\ z \in C}} \operatorname{dist}(z, \partial B) / \mathfrak{R} z=1 \text {. }
$$

Denote $q_{z}=\operatorname{dist}(z, \partial B), V_{z}=\left\{y \in \mathbb{C}:|z-y|<q_{z}\right\}$ and $V=\{y \in \mathbb{C}:$ $|y|<1\}$. For each $z \in C$ with $q_{z}>0$ define a function $f_{z}: V \rightarrow \mathbb{C}$ by 
$f_{z}(y)=f\left(q_{z} y+z\right) / \Re z$.

Fix some $\varepsilon>0$. Choose $z \in C$ so close to 0 that

$$
b-\varepsilon \leq \Re f(y) / \Re y \leq b+\varepsilon
$$

for all $y \in V_{z}$ (see (3.4)) and $1-\varepsilon \leq q_{z} / \mathfrak{R} z \leq 1+\varepsilon$. Then for $y \in V$,

$$
\begin{aligned}
\Re f_{z}(y) & =\Re f\left(q_{z} y+z\right) / \Re z \\
& \leq(b+\varepsilon) \Re\left(q_{z} y+z\right) / \Re z \\
& \leq(b+\varepsilon)\left(\Re y q_{z} / \Re z+1\right) \\
& \leq(b+\varepsilon)(\Re y(1+\varepsilon)+1) .
\end{aligned}
$$

Analogously,

$$
\Re f_{z}(y) \geq(b-\varepsilon)(\Re y(1-\varepsilon)+1) .
$$

Thus the family $\left\{\Re f_{z}(y)\right\}$ of positive harmonic functions converges uniformly on $V$ to $b(\Re y+1)$ as $z \rightarrow 0, z \in C$. An easy variation of the Harnack's Convergence Theorem (Doob (1984) 1 II 3(a) and (b)) shows that the first partial derivatives of $\Re f_{z}(y)$ converge uniformly on compact subsets of $V$ to those of $b(\Re y+1)$. It follows that

Notice that

$$
\lim _{\substack{z \rightarrow 0 \\ z \in C}} f_{z}^{\prime}(0)=b \text {. }
$$

$$
f(y)=f_{z}\left((y-z) / q_{z}\right) \Re z
$$

for $(y-z) / q_{z} \in V$ and, therefore,

$$
f^{\prime}(y)=f_{z}^{\prime}\left((y-z) / q_{z}\right) \Re z / q_{z}
$$

and

$$
f^{\prime}(z)=f_{z}^{\prime}(0) \Re z / q_{z} .
$$

In view of (3.6), this implies that $\lim _{z \rightarrow 0} f^{\prime}(z)=b$. The set $C$ is a minimal $\underset{\substack{z \rightarrow 0 \\ z \in C}}{z \in C}$

fine neighborhood of 0 in $D_{*}$, by (3.5) and Lemma 2.1. It follows that mf- $f^{\prime}(0)=b \in \mathbb{R}_{+}$.

CoRollary 3.1. Suppose that $f_{1}: D_{*} \rightarrow D_{1} \subset D_{*}, f_{2}: D_{*} \rightarrow D_{2}, f_{1}(0)=0$ and $\mathrm{mf}-f_{k}^{\prime}(0) \in \mathbb{R}_{+}$for $k=1,2$. Then $\mathrm{mf}-\left(f_{2} \circ f_{1}\right)^{\prime}(0) \in \mathbb{R}_{+}$.

Proof. Let $A_{k}$ be a minimal fine neighborhood of 0 in $D_{*}$ such that $\lim _{\substack{z \rightarrow 0 \\ z \rightarrow A_{k}}} f_{k}^{\prime}(z)=m f-f_{k}^{\prime}(0)$, for $k=1,2$. Such sets $A_{k}$ exist by Theorem 1 XII 16 of Doob (1984). The set $D_{1}$ is a minimal fine neighborhood of 0 in $D_{*}$, 
by Theorem 3.2. Therorem 15 of Naim (1957) shows that $A_{3} \stackrel{\text { df }}{=} A_{2} \cap D_{1}$ is a minimal fine neighborhood of $0^{M}$ in $D_{1}$. By the conformal invariance of the minimal fine topology, the sets $A_{4} \stackrel{\text { df }}{=} f_{1}^{-1}\left(A_{3}\right)$ and $A_{5} \stackrel{\text { df }}{=} A_{4} \cap A_{1}$ are minimal fine neighborhoods of 0 in $D_{*}$. Now it is easy to see that

$$
\begin{aligned}
\lim _{\substack{z \rightarrow 0 \\
z \in A_{5}}}\left(f_{2} \circ f_{1}\right)^{\prime}(z) & =\lim _{\substack{z \rightarrow 0 \\
z \in A_{5}}} f_{2}^{\prime}\left(f_{1}(z)\right) f_{1}^{\prime}(z) \\
& =\mathrm{mf}-f_{2}^{\prime}(0) \cdot \mathrm{mf}-f_{1}^{\prime}(0)
\end{aligned}
$$

and this completes the proof.

Corollary 3.2. Suppose that $f_{k}: D_{*} \rightarrow D$ and $\operatorname{mf}-\lim _{\substack{z \rightarrow 0 \\ z \in D_{*}}} f_{k}(z)=0^{M} \in$ $\partial_{1}^{M} D$ for $k=1,2$. If $\mathrm{mf}-f_{1}^{\prime}(0) \in \mathbb{R}_{+}$then $\mathrm{mf}-f_{2}^{\prime}(0) \in \mathbb{R}_{+}$.

Proof. The function $f_{3} \stackrel{\text { df }}{=} f_{1}^{-1} \circ f_{2}$ maps $D_{*}$ onto $D_{*}$ and $f_{3}(0)=0$ so by Theorem 10.2 of Pommerenke (1975), mf- $f_{3}^{\prime}(0) \in \mathbb{R}_{+}$. Corollary 3.1 implies that

$$
\operatorname{mf}-f_{2}^{\prime}(0)=\operatorname{mf}-\left(f_{1} \circ f_{3}\right)^{\prime}(0) \in \mathbb{R}_{+} .
$$

Corollary 3.3. Suppose that $D_{1} \subset D_{2}$ and $0^{M} \in \partial_{1}^{M} D_{2}$ is well defined. Assume that $D_{k}$ has a minimal fine derivative $(k=1$ or 2$)$. Then $D_{3-k}$ has minimal fine derivative if and only if $D_{2} \backslash D_{1}$ is minimal thin in $D_{2}$ at $0^{M}$.

Proof. i) If $D_{1}$ and $D_{2}$ have minimal fine derivatives then they have angular derivatives and $D_{2} \backslash D_{1}$ is minimal thin in $D_{2}$ at $0^{M}$, by Corollary 9.1 of Burdzy (1987).

ii) Suppose that $D_{2}$ has a minimal fine derivative and $D_{2} \backslash D_{1}$ is minimal thin in $D_{2}$ at $0^{M}$. Let $f_{2}: D_{*} \rightarrow D_{2}, f_{2}(0)=0$, mf- $f_{2}^{\prime}(0) \in \mathbb{R}_{+}$. The set $f_{2}^{-1}\left(D_{1}\right)$ is a minimal fine neighborhood of 0 in $D_{*}$, by the conformal invariance of the minimal fine topology. Thus $f_{2}^{-1}\left(D_{1}\right)$ has a minimal fine derivative, by Theorem 3.2. Let

$$
f_{3}: D_{*} \rightarrow f_{2}^{-1}\left(D_{1}\right), \quad f_{3}(0)=0, \quad \operatorname{mf}-f_{3}^{\prime}(0) \in \mathbb{R}_{+}
$$

and $f_{1} \stackrel{\text { df }}{=} f_{2} \circ f_{3}$. Then $f_{1}: D_{*} \rightarrow D_{1}, f_{1}(0)=0$ and

$$
\operatorname{mf}-f_{1}^{\prime}(0)=\operatorname{mf}-\left(f_{2} \circ f_{3}\right)^{\prime}(0) \in \mathbb{R}_{+},
$$

by Corollary 3.1. It may be concluded that $D_{1}$ has a minimal fine derivative.

iii) Assume that $D_{1}$ has a minimal fine derivative and $D_{2} \backslash D_{1}$ is mini- 
mal thin in $D_{2}$ at $0^{M}$. Choose functions $f_{1}$ and $f_{2}$ so that $f_{1}: D_{*} \rightarrow D_{1}$, $f_{1}(0)=0, \operatorname{mf}-\lim _{\substack{z \rightarrow 0 \\ z \in D_{*}}} f(z)=0^{M}$ and let $f_{3} \stackrel{\text { df }}{=} f_{2}^{-1} \circ f_{1}$. Then $f_{3}: D_{*} \rightarrow f_{2}^{-1}\left(D_{1}\right)$ and mf- $\lim _{z \rightarrow 0} f_{3}(z)=0^{M}$. The set $f_{2}^{-1}\left(D_{1}\right)$ is a minimal fine neighborhood of 0 in $D_{*}$, by the conformal invariance of such neighborhoods. It follows that $f_{2}^{-1}\left(D_{1}\right)$ has a minimal fine derivative (Theorem 3.2) and mf- $f_{3}^{\prime}(0) \in \mathbb{R}_{+}$ (Corollary 3.2). Let $A_{1}$ and $A_{3}$ be minimal fine neighborhoods of 0 in $D_{*}$ such that $\lim _{\substack{z \rightarrow 0 \\ z \in A_{k}}} f_{k}^{\prime}(z)=\operatorname{mf}-f_{k}^{\prime}(0)$ for $k=1,3$ (see Theorem 1 XII 16 of Doob (1984)). Define $A_{2}=A_{1} \cap A_{3}$ and $A=f_{3}\left(A_{2}\right)$. The set $A$ is a minimal fine neighborhood of $0^{M}$ in $f_{2}^{-1}\left(D_{1}\right)$, by conforml invariance. Theorem 15 of Naim (1957) implies that this set is a minimal fine neighborhood of 0 in $D_{*}$ as well. One has

$$
\begin{aligned}
\lim _{\substack{z \rightarrow 0 \\
z \in A}} f_{2}^{\prime}(z) & =\lim _{\substack{z \rightarrow 0 \\
z \in A}}\left(f_{1} \circ f_{3}^{-1}\right)^{\prime}(z) \\
& =\lim _{\substack{z \rightarrow 0 \\
z \in A}} f_{1}^{\prime}\left(f_{3}^{-1}(z)\right)\left(f_{3}^{-1}\right)^{\prime}(z) \\
& =\operatorname{mf}-f_{1}^{\prime}(0) \times \operatorname{mf}-f_{3}(0) \in \mathbb{R}_{+}
\end{aligned}
$$

and it follows from Theorem 1 XII 16 of Doob (1984) that $m f-f_{2}^{\prime}(0) \in \mathbb{R}_{+}$. Thus $D_{2}$ has a minimal fine derivative.

Corollary 3.4. If $D_{1} \subset D_{2} \subset D_{3}$ and $D_{1}$ and $D_{3}$ have minimal fine derivatives then so does $D_{2}$.

Proof. By Corollary 3.3, $D_{3} \backslash D_{1}$ is minimal thin in $D_{3}$ at $0^{M} \in \partial_{1}^{M} D_{3}$ and the same may be said about its subset $D_{3} \backslash D_{2}$. Thus $D_{2}$ has a minimal fine derivative, by the same Corollary 3.3.

For a domain $D$, let $W_{\varepsilon, D}$ denote the smallest Lipschitz function $g$ : $\mathbb{R} \rightarrow \mathbb{R}$ such that

$$
\{z \in \mathbb{C}: z \notin D,|z| \leq \varepsilon\} \subset\{z \in \mathbb{C}: \Re z \leq g(\mathfrak{\Im} z)\} .
$$

Theorem 3.3. i) Suppose that $0 \in \partial D$ and for some $\varepsilon>0$

$$
\int_{-1}^{1} \max \left(W_{\varepsilon, D}(r), 0\right) r^{-2} d r<\infty
$$

Then the domain $D$ has a minimal fine derivative if and only if

$$
\int_{-1}^{1} \min \left(W_{\varepsilon, D}(r), 0\right) r^{-2} d r>-\infty
$$


ii) Suppose that $0 \in \partial D$ and let $D_{1}=D \cup D_{*}$. Assume that

$$
\int_{-1}^{1} \min \left(W_{\varepsilon, D_{1}}(r), 0\right) r^{-2} d r>-\infty
$$

for some $\varepsilon>0$. Then $D$ has a minimal fine derivative if and only if

$$
\int_{-1}^{1} \max \left(W_{\varepsilon_{1}, D}(r), 0\right) r^{-2} d r<\infty
$$

for some $\varepsilon_{1}>0$.

Proof. Corollary 3.3 and Theorem 3.3 are true when "minimal fine derivative" is replaced by "angular derivative" in their statements. This has been proved in Corollary 9.1 and Theorems 9.3 and 9.4 of Burdzy (1987) and will be applied here to give a quick poof of the theorem.

i) Assume (3.7). Theorem 9.3 of Burdzy (1987) shows that $D \cap D_{*}$ has an angular derivative which in turn implies that $D_{*} \backslash D$ is minimal thin in $D_{*}$ at 0 , by Corollary 9.1 of Burdzy (1987). It follows from Theorem 3.2 that $D \cap D_{*}$ has a minimal fine derivative. $D$ has an angular derivative if and only if (3.8) holds, by Theorem 9.3 of Burdzy (1987). Thus (3.8) is equivalent to minimal thinness of $D \backslash\left(D \cap D_{*}\right)$ in $D$ at $0^{M}$, by Corollary 9.1 of Burdzy (1987). It remains to use Corollary 3.3 to see that (3.8) holds if and only if $D$ has a minimal fine derivative.

ii) This part may be proved in a completely analogous way.

Definition 3.3. Consider a function $f: D_{*} \rightarrow D$. A point $x \in \partial D_{*}$ will be called an $f$-twist point if $f(x)$ exists and for every continuous path $\{\Gamma(t), t \in(0,1)\}$ in $D_{*}$ with $\lim _{t \rightarrow 0} \Gamma(t)=x$, one has

$$
-\liminf _{t \rightarrow 0} \arg (f(\Gamma(t))-f(x))=\limsup _{t \rightarrow 0} \arg (f(\Gamma(t))-f(x))=\infty .
$$

Theorem 3.4. Suppose that $f: D_{*} \rightarrow D$. Then for almost all $x \in \partial D_{*}$, either $\mathrm{mf}-f^{\prime}(x)$ exists and belongs to $\mathbb{C} \backslash\{0\}$ or $x$ is an $f$-twist point.

Proof. Consult the next section for the probabilistic notation used in this proof.

It is well known that $f(x)$ exists for almost all $x \in \partial D_{*}$.

Fix some $y \in D_{*}$. The arguments given in Section 4 of Davis (1979) or Section 5.4 of Durrett (1984) show that for almost every $x \in \partial D_{*}$ either

i) $\lim _{t \rightarrow R} f^{\prime}\left(X_{t}\right)$ exists and belongs to $\mathbb{C} \backslash\{0\} P_{x}^{y}$-a.s. ( $P_{x}^{y}$ is the distribution of the $h$-process in $D_{*}$ starting from $y$ and converging to $x$ at its lifetime $R$ ) or 
ii) $f(S(\alpha)+x)$ is dense in $\mathbb{C}$ for all $\alpha<\pi / 2$.

In the first case, mf- $f^{\prime}(x)=\operatorname{mf}-\lim _{z \rightarrow x} f^{\prime}(z)$ exists and belongs to $\mathbb{C} \backslash\{0\}$, by Theorem 3 III 3 of Doob (1984).

Lemmas 10.8 and 10.9 of Pommerenke (1975) prove that almost all points satisfying (ii) are $f$-twist points.

Remarks 3.3. i) Theorem 3.4 is a slight generalization of McMillan's Theorem (Pommerenke (1975), Theorem 10.15).

ii) Theorems 3.1-3.4 and Corollaries 3.1-3.4 remain true when minimal fine limits and derivatives are replaced by angular limits and derivatives in their statements (see Burdzy (1986, 1987), Jackson (1965) and Pommerenke (1975)). They are among the strongest results about angular derivatives available now.

\section{§4. Brownian excursions}

A detailed review of probabilistic concepts presented here may be found in Doob (1984) and Burdzy (1987).

The canonical stochastic process will be denoted $X$ i.e., $X_{t}(\omega)=\omega(t)$ for $t>0$ and $\omega \in \Omega . \quad \Omega$ is the family of all paths $\omega: \mathbb{R}_{+} \rightarrow \mathbb{C} \cup\{\delta\}$, continuous on $(0, R)$ and equal to $\delta$ afterwards. Thus $R$ is the lifetime of $X$. The point $\delta$ is a "coffin" state outside $\mathbb{C}$. The distribution and the corresponding expectation of the standard Brownian motion in $\mathbb{C}$ starting from $x$ will be denoted $P^{x}$ and $E^{x}$. The symbol $P_{D}^{x}$ will stand for the distribution of Brownian motion in a domain $D$ i.e., Brownian motion killed at the hitting time $T_{D^{c}}$ of $D^{c}, T_{D^{c}} \stackrel{\text { df }}{=} \inf \left\{t>0: X_{t} \notin D\right\}$. The distribution of an $h$-process in $D$ starting from $x$ and converging to $y \in \partial_{1}^{M} D$ at its lifetime in the Martin topology will be denoted $P_{y}^{x}$. This symbol has been used in Section 3 and will not be used any more.

For a domain $D$ and $x$ in $\partial D$ or $\partial_{1}^{M} D$, a standard excursion law $H^{x}$ in $D$ is a $\sigma$-finite measure on $\Omega$ such that

i) $X$ is strong Markov with respect to $P_{D}^{x}$ transition probabilities,

ii) $H^{x}\left(\lim _{t \rightarrow 0} X_{t} \neq x\right)=0$ where the limit is taken in the Euclidean or Martin topology, depending on whether $x \in \partial D$ or $x \in \partial_{1}^{M} D$,

iii) $0<H^{x}\left(T_{B}<\infty\right)<\infty$ for all compact nonpolar sets $B \subset D$.

If $x \in \partial_{1}^{M} D$ and a standard excursion law $H^{x}$ in $D$ exists then it is unique up to a multiplicative constant.

A prime end $x \in \partial_{1}^{M} D$ will be called accessible if there exists a con- 
tinuous path in $D$ which converges to $x$ in the Martin topology and to some $y \in \mathbb{C}$ in the Euclidean topology. If $x \in \partial_{1}^{M} D$ is accessible then the standard excursion law $H^{x}$ in $D$ exists.

For $t>0$ such that $X(t) \in \partial D$ define excursions in $D$ as

$$
e_{t}(s)= \begin{cases}X(t+s) & \text { if } \inf \left\{u>t: X_{u} \notin D\right\}>t+s, \\ \delta & \text { otherwise. }\end{cases}
$$

The excursion $e_{t}(s)$ is defined for $s>0$ and the possibility $e(\cdot) \equiv \delta$ is not excluded. Let $L_{t}$ be the local time of Brownian motion on $\partial D$. Then there exists a family $\left\{H^{x}, x \in \mathbb{C}\right\}$ of $\sigma$-finite measures such that

$$
E^{\cdot}\left(\sum_{0<u<\infty} Z_{u}(f(e))=E^{\cdot}\left(\int_{0}^{\infty} Z_{s} H^{X_{s}}(f) d L_{s}\right)\right.
$$

for all predictable processes $Z$ and universally measurable $f$ which vanish on excursions constantly equal to $\delta$ (Maisonneuve (1975)). The measures $H^{x}$ may be chosen so that each $H^{x}$ is either null or it is a standard excursion law in $D$. Every pair $(d L, H)$ satisfying (4.1) will be called an exist system in $D$.

Consider a function $f: D_{1} \rightarrow D_{2}$, an accessible prime end $x \in \partial_{1}^{M} D_{1}$ and a standard excursion law $H^{x}$ in $D_{1}$. Put $f^{\prime}(y)=0$ for $y \notin D_{1}$ and define

$$
c(t)=\int_{0}^{t}\left|f^{\prime}(X(s))\right|^{2} d s \quad \text { for } t \in(0, R)
$$

and

$$
Y(c(t))= \begin{cases}f(X(t)) & \text { if } t \in(0, R) \text { and } c(t)>\infty, \\ \delta & \text { otherwise. }\end{cases}
$$

If $X$ has the distribution $H^{x}$ and $f$ maps $x$ onto an accessible prime end in $D_{2}$ then $c(t)<\infty$ and the distribution of $Y$ is a standard excursion law in $D_{2}$, denoted $f\left(H^{x}\right)$.

Definition 4.1. Consider a domain $D, x \in \partial D$ and an excursion law $H^{x}$ in $D . H^{x}$ will be called

i) almost locally flat or

ii) a twist excursion law

if there exists a function $f: D_{*} \rightarrow D$ and a standard excursion law $H^{y}$ in $D_{*}$ such that $H^{\times}=f\left(H^{y}\right)$ and

i) $\operatorname{mf}-f^{\prime}(y) \in \mathbb{C} \backslash\{0\}$, 
ii) $y$ is an $f$-twist point.

Remark 4.1. See Burdzy (1987) for the comparison and explanation of the terminology.

Theorem 4.1. For each domain $D$ there exists an exit system $(d L, H)$ in $D$ such that all measures $H^{x}$ are either null measures, twist excursion laws, almost locally flat excursion laws or mixtures of such measures.

Proof. The proof is completely analogous to the proof of Theorem 10.1 of Burdzy (1987) and therefore it will be only sketched here.

Let $f: D_{*} \rightarrow D$ and for each $x \in \partial D_{*}$ let $H_{1}^{x}$ be the standard excursion law in $D_{*}$. For almost all $x \in \partial D_{*}, \operatorname{mf}-\lim _{z \rightarrow x} f(z)$ is an accessible prime end and $f\left(H_{1}^{x}\right)$ is a twist or almost locally flat excursion law in $D$, by Theorem 3.4. By the uniqueness of the standard excursion laws and conformal invariance of the Martin boundary, almost all standard excursion laws in $D$ (starting from points of $\partial_{1}^{M} D$ ) are either twist or almost locally flat. This essentially completes the proof.

Theorem 4.2. Consider a domain $D, x \in \partial D$ and an almost locally fiat excursion law $H^{x}$ in $D$. Then

i) $\lim _{t \rightarrow 0} \inf \arg \left(X_{t}-x\right)=\limsup _{t \rightarrow 0} \arg \left(X_{t}-x\right)-\pi \quad H^{x}$-a.e. and

ii) $\limsup _{t \rightarrow 0} \frac{\left|X_{t}-x\right|}{\sqrt{2 t \log \log (1 / t)}}=1 \quad H^{x}$-a.e.

Proof. Let $f: D_{*} \rightarrow D$ and $y \in \partial D_{*}$ be such that mf- $f^{\prime}(y)=a \in \mathbb{C} \backslash\{0\}$ and $H^{x}=f\left(H^{y}\right)$ where $H^{y}$ is the standard excursion law in $D_{*}$. Observe that necessarily $f(y)=x$.

i) $X_{t}$ converges to $y$ in the minimal fine topology $H^{y}$-a.e. (Burdzy (1987) Remark 3.4 (i)) so

$$
\lim _{t \rightarrow 0} f^{\prime}\left(X_{t}\right)=\lim _{t \rightarrow 0}\left(f\left(X_{t}\right)-x\right) /\left(X_{t}-y\right)=a \neq 0
$$

$H^{y}$-a.e., by Theorem 3.1. Remark 5.2 (iii) of Burdzy (1987) implies that

$$
\liminf _{t \rightarrow 0} \arg \left(X_{t}-y\right)=\limsup _{t \rightarrow 0} \arg \left(X_{t}-y\right)-\pi
$$

$H^{y}$-a.e. and this combined with (4.3) shows that

$$
\liminf _{t \rightarrow 0} \arg \left(f\left(X_{t}\right)-x\right)=\limsup _{t \rightarrow 0} \arg \left(f\left(X_{t}\right)-x\right)-\pi
$$

$H^{y}$-a.e. This is equivalent to the assertion (i). 
ii) (4.3) implies that

$$
\lim _{t \rightarrow 0}\left|f\left(X_{t}\right)-x\right| /\left|X_{t}-y\right|=|a| \quad H^{y} \text {-a.e. }
$$

Since $X_{t}$ converges to $y$ in the minimal fine topology $H^{y}$-a.e.,

$$
\lim _{t \rightarrow 0} f^{\prime}\left(X_{t}\right)=a \quad H^{y} \text {-a.e. }
$$

and, by (4.2),

$$
\lim _{t \rightarrow 0} c(t) / t=a \quad H^{y} \text {-a.e. }
$$

The following Law of Iterated Logarithm holds for $H^{y}$ (Burdzy (1987) Remark 5.2 (ii)).

$$
\limsup _{t \rightarrow 0} \frac{\left|X_{t}-y\right|}{\sqrt{2 t \log \log (1 / t)}}=1 \quad H^{y} \text {-a.e. }
$$

Formulae (4.4)-(4.6) yield

$$
\begin{aligned}
\limsup _{t \rightarrow 0} & \frac{\left|f\left(X_{t}\right)-x\right| /|a|}{\sqrt{2(c(t) /|a|)^{2} \log \log \left(|a|^{2} / c(t)\right)}} \\
& =\limsup _{t \rightarrow 0} \frac{\left|f\left(X_{t}\right)-x\right|}{\sqrt{2 c(t) \log \log (1 / c(t))}}=1 \quad H^{y} \text {-a.e. }
\end{aligned}
$$

which is equivalent to the second assertion.

Corollary 4.1. Suppose that an exit system $(d L, H)$ in a domain $D$ does not contain any twist excursion laws. Then for all $x \in \mathbb{C}, P^{x}$-a.s. all nonconstant excursions $\left\{e_{t}(s), s>0\right\}$ of Brownian motion in $D$ have the following properties.

i) $\liminf _{s \rightarrow 0} \arg \left(e_{t}(s)-e_{t}(0+)\right)=\limsup _{s \rightarrow 0} \arg \left(e_{t}(s)-e_{t}(0+)\right)-\pi$ and

ii) $\limsup _{s \rightarrow 0} \frac{\left|e_{t}(s)-e_{t}(0+)\right|}{\sqrt{2 s \log \log (1 / s)}}=1$.

Proof. The result follows immediately from the exit system formula (4.1) and Theorems 4.1 and 4.2.

Remarks 4.2. i) Twist excursion laws may be precluded by the shape of the domain $D$. For example, there are no twist excursions in Lipschitz domains, starshaped domains etc.

ii) If $H^{x}$ is almost locally flat, it need not be true that the range of $\left\{\arg \left(X_{t}-x\right), 0<t<\varepsilon\right\}$ is an interval of length $\pi$ (open or closed) $H^{x}$ - 
-a.e., even for small $\varepsilon>0$. Proposition 3.1 of Burdzy and Williams (1986) presents a $C^{1}$-domain such that the range of $\left\{\arg \left(e_{t}(s)-e_{t}(0+)\right), 0<s<\right.$ $\varepsilon\}$ is an interval of length greater than $\pi$ for every nonconstant excursion $e_{t}$, every $\varepsilon>0, P^{x}$-a.s. for all $x \in \mathbb{C}$.

\section{REFERENCES}

[1] Burdzy, K., Brownian excursions and minimal thinness, Part III, Applications to the angular derivative problem, Math. Z., 192 (1986), 89-107.

[2] - "Multidimensional Brownian Excursions and Potential Theory," Longman London (1987).

[ 3 ] Burdzy, K. and Williams, R. J., On Brownian escursions in Lipschitz domains, Part I, Local path properties, Trans. Amer. Math. Soc., 298 (1986), 289-306.

[ 4 ] Davis, B., Brownian motion and analytic functions, Ann. Probab., 7 (1979), 913-932.

[5] Doob, J. L., "Classical Potential Theory and Its Probabilistic Counterpart," Springer, New York (1984).

[6] Durrett, R., "Brownian Motion and Martingales in Analysis," Wadsworth, Belmont (1984).

[ 7 ] Essén, M. and Jackson, H. L., On the covering properties of certain exceptional sets in a halfspace, Hiroshima Math. J., 10 (1980), 233-262.

[8] Jackson, H. L., Some remarks on angular derivatives and Julia's Lemma, Can. Math. Bull., 9 (1965), 233-241.

[9] - On the boundary behaviour of BLD functions and some applications, Bull. Cl. Sc. Acad. R. Belgique, 66 (1980), 223-239.

[10] Maisonneuve, B., Exit systems, Ann. Probab., 3 (1975), 399-411.

[11] Naim, L., Sur le rôle de la frontière de R. S. Martin dans la théorie du potential, Ann. Inst. Fouriere, Grenoble, 7 (1957), 183-281.

[12] Pommerenke, C., "Univalent Functions," Vandenhoeck and Ruprecht, Göttingen, (1975).

[13] Rodin, B. and Warschawski, S. E., Extremal length and univalent functions. I. The angular derivative, Math. Z., 153 (1977), 1-17.

Department of Mathematics

University of Washington

Seattle, WA 98195

U.S.A. 